\title{
Lesión renal aguda en COVID-19. Análisis en el Hospital Ángeles Mocel
}

\section{Acute kidney injury in COVID-19. Analysis at the Hospital Ángeles Mocel}

\author{
Pamela Michelle Prado Lozano, ${ }^{*}$ Fernando Cortés Colula, ${ }^{*}$ \\ Mariana Delgado Nava, * Antonio Fernández Macías Valadez, * Andrea Magdalena Luna Hernández, * \\ Mayte Mesinas Garrido, * Francisco Velasco García Lascurain,* María de los Ángeles Villeda Aguilar,* \\ Daniel Fernando Ovando Morga ${ }^{\ddagger}$
}

Citar como: Prado LPM, Cortés CF, Delgado NM, Fernández MVA, Luna HAM, Mesinas GM et al. Lesión renal aguda en COVID-19. Análisis en el Hospital Ángeles Mocel. Acta Med Grupo Angeles. 2021; 19 (2): 236-243. https://dx.doi.org/10.35366/100448

\section{Resumen}

Introducción: La lesión renal aguda (LRA) es común en enfermos con COVID-19. Sin embargo, su incidencia actual se basa en series de casos y estudios retrospectivos, que reportan un rango muy amplio de presentación. Se ha sugerido que la LRA es un factor de riesgo independiente para el requerimiento de ventilación mecánica y muerte intrahospitalaria en pacientes con dicha enfermedad viral. Material y métodos: Estudio observacional, descriptivo, transversal y retrospectivo. Se revisaron los expedientes de enfermos internados en el Hospital Ángeles Mocel con diagnóstico de COVID-19, entre marzo y julio del 2020. Se incluyeron personas mayores de edad, con diagnóstico confirmado de esa infección y expediente clínico completo $(n=83)$. Se utilizó estadística descriptiva a fin de calcular porcentajes, medias y desviación estándar. Resultados: El 36.14\% desarrolló LRA, 43.3\% fueron nivel uno de KDIGO (Kidney Disease: Improving Global Outcomes), 20\% en KDIGO 2, 36.6\% KDIGO 3 y $23.3 \%$ requirieron terapia de reemplazo de riñón. De quienes tenían ventilación mecánica invasiva (VMI), 77.7\% generaron LRA. El 56.6\% de aquellos con LRA fallecieron. Conclusiones: La incidencia de LRA en afectados con COVID-19 en esta población fue similar a lo reportado en la literatura internacional. Fue más frecuente en pacientes en unidad de cuidados intensivos y con VMI, en quienes se observó más alta mortalidad.

Palabras clave: COVID-19, SARS-CoV-2, coronavirus, lesión renal aguda, hemodiálisis.

\footnotetext{
* Servicio de Medicina Interna, Hospital Ángeles Mocel. Facultad Mexicana de Medicina, Universidad La Salle México.

₹ Servicio de Nefrología, Centro Médico Nacional 20 de Noviembre.
}

Aceptado: 05-05-2021.

Correspondencia:

Pamela Michelle Prado Lozano

Correo electrónico: pam.prado.lozano@gmail.com

www.medigraphic.com/actamedica

\section{Abstract}

Introduction: Acute kidney injury (AKI) is common in COVID-19 patients. Nonetheless, its actual incidence is based in case series and retrospective studies, reporting a wide range of presentations. It has been suggested that AKI is an independent risk factor for mechanical ventilation and death in patients with that viral infection. Material and methods: Observational, descriptive, cross-sectional and retrospective study. The files of interned patients at Hospital Ángeles Mocel between March and July 2020, with a COVID-19 diagnosis, were reviewed. Patients over 18 years old, with a confirmed COVID-19 diagnosis and a complete file were included $(n=$ 83). A descriptive analysis to calculate percentages, means and standard deviation was employed. Results: $36.14 \%$ of patients developed $\mathrm{AKI}, 43.3 \%$ were classified as KDIGO 1 , $20 \%$ as KDIGO 2, $36.6 \%$ as KDIGO 3, and $23.3 \%$ required kidney replacement therapy. Of the patients under invasive mechanical ventilation, $77.7 \%$ developed AKI. Out of the total of patients with AKI, $56.6 \%$ died. Conclusions: The incidence of AKI in COVID-19 patients in our population, was similar to that reported in the international literature. AKI was more frequent in patients hospitalized in intensive care units and under invasive mechanical ventilation, in which a higher mortality was observed.

Keywords: COVID-19, SARS-CoV-2, coronavirus, acute kidney injury, hemodialysis.

\section{INTRODUCCIÓN}

A finales de diciembre de 2019 surgieron una serie de casos de neumonía de etiología desconocida en la provincia de Hubei, China, cuya presentación era similar a una neumonía viral. Gracias a la secuenciación de muestras del tracto respiratorio superior de estos pacientes logró aislarse un nuevo coronavirus, Ilamado SARS-CoV-2, responsable de la enfermedad por coronavirus 2019 
o COVID-19, por sus siglas en inglés y causante de la pandemia actual. ${ }^{1,2}$

Dicha pandemia ha evolucionado rápidamente y con ello su espectro clínico se ha hecho cada vez más evidente. Desde una forma leve y autolimitada hasta el síndrome de insuficiencia respiratoria aguda (SIRA), falla multiorgánica y muerte. ${ }^{3}$

Aunque esta enfermedad afecta principalmente el tracto respiratorio, el riñón es otro de sus blancos. La lesión renal aguda (LRA) es común en contagiados con el citado coronavirus, particularmente en aquellos con infecciones severas, y se considera un marcador de mayor morbimortalidad. ${ }^{3-5}$

Dentro de los factores de riesgo hacia el desarrollo de LRA en COVID-19 descritos hasta ahora se encuentran: antecedente de hipertensión arterial, insuficiencia cardiaca, enfermedad crónica de riñón, uso de medicamentos nefrotóxicos, reanimación intravenosa subóptima por el riesgo de deterioro respiratorio, una presión positiva al final de la espiración (PEEP) alta que puede disminuir el retorno venoso, la intubación prolongada, el estado proinflamatorio y protrombótico. ${ }^{6,7}$

Un estudio monocéntrico retrospectivo realizado en Nueva York reportó más elevada aparición de LRA asociada a COVID-19 en los adultos mayores de 60 años, contrastado con personas más jóvenes (84 versus 16\%). En esta investigación también hubo incidencia aumentada de insuficiencia cardiaca (19 versus 4\%), hipertensión (80 contra $64 \%$ ) y ERC (27 versus $9 \%$ ) en aquellos con LRA, comparado con quienes no generaron afección renal. Además, al ingreso, quienes padecían LRA tenían requerimientos de oxígeno más altos $\left(\mathrm{FiO}_{2} 44\right.$ versus $\left.34 \%\right)$ y niveles de dímero D más elevados $(5,468$ contra 2,248$){ }^{8}$

El surgimiento de LRA en COVID-19 se basa actualmente sólo en informes de series de casos y estudios retrospectivos. Reportes de China, Europa y EUA han indicado un rango muy amplio de aparición, desde el 1 hasta 42\%.6,9 Guan y colaboradores reportaron una incidencia de $0.5 \%$ en 1,099 pacientes de 552 hospitales en China. Mientras que un artículo irlandés reportó que de 3,908 enfermos admitidos a la Unidad de Cuidados Intensivos (UCl), 22.2\% desarrollaron LRA que necesitó terapia de reemplazo renal (TRR) con hemodiálisis convencional, con una mortalidad superior al 75\%. En una cohorte de 99 afectados críticos con SARS-CoV-2, Gabarre y colegas reportaron desencadenamiento de LRA en $42.9 \%$ de los involucrados, de los cuales $74.4 \%$ fueron clasificados con el grado tres de acuerdo a la definición de las guías Kidney Disease Improving Global Outcomes (KDIGO) ${ }^{10}$ y $13.4 \%$ requirió TRR. ${ }^{6,9}$

Por otro lado, un metaanálisis que incluyó 6,945 casos, de China, Italia, Reino Unido y EUA, encontró una presentación de LRA en contagiados con este coronavirus de 8.9\% (IC 95\% 4.6-14.5). ${ }^{11}$
Los estudios comentados anteriormente sugieren que la LRA es un factor de riesgo independiente para el deterioro clínico y muerte intrahospitalaria en infectados con SARSCoV-2. Estos pacientes son más propensos a requerir manejo en $\mathrm{UCl}$, ventilación asistida y uso de vasopresores. $7,8,12$

La mortandad hospitalaria en afectados con COVID-19 y LRA asociada puede alcanzar hasta el $45 \%$, comparada con $7 \%$ en aquellos sin LRA asociada $(p<0.001) .{ }^{9}$ Así mismo, existe una letalidad marcadamente incrementada en dichos pacientes que generaron LRA en UCI (52\%) y fuera de $\mathrm{UCI}(41 \%)$, contrastado con otros sin LRA ( 9 y $7 \%$, respectivamente). ${ }^{9}$

En otro artículo, publicado por Chan y asociados, los pacientes con LRA tuvieron mayor riesgo de mortalidad con un OR 9.6 (IC 95\% 7.4-12.3), cotejado con quienes no presentaron LRA, posterior al análisis multivariado. ${ }^{9}$

En cuanto a los pacientes hospitalizados en $\mathrm{UCl}$, quienes padecían LRA presentaron un OR 20.9 (IC 95\% 11.7-37.3) de mortandad, comparado con los otros sin LRA. En este trabajo no se halló correlación con comorbilidades como hipertensión arterial sistémica (HAS) y diabetes mellitus tipo dos (DM2) con LRA severa. Sin embargo, la enfermedad renal crónica (ERC) si se asoció de manera independiente. ${ }^{9}$

Una revisión sistemática y metaanálisis explorando la supervivencia en contagiados con SARS-CoV-2 que desarrollaron LRA severa, definida en el estadio tres de KDIGO, encontró una asociación positiva entre ésta y el aumento de la mortalidad con un RR 4.19 (IC 95\% 3.31-5.31). ${ }^{13}$ Mientras que otro estudio que incluyó 5,449 pacientes, de una red de 13 hospitales en EUA, reportó que 35\% de los enfermos que generaron LRA fallecieron. De éstos, 34\% presentaron LRA estadio uno de KDIGO, 64\% estadio dos y $91 \%$ estadio tres. Del total de enfermos que requirieron TRR, el 55\% fallecieron. Entre aquellos que sobrevivieron y fueron dados de alta, la mediana del pico máximo de creatinina sérica fue de $2.34 \mathrm{mg} / \mathrm{dL}$ (rango inter cuartil (RIC): $1.42-4.25 \mathrm{mg} / \mathrm{dL}$ ). Por otra parte, en el grupo de quienes necesitaron ventilación artificial, 65.5\% presentaron LRA, en contraste con $6.7 \%$ de los que no la recibieron. Además, $96.8 \%$ de los internados que precisaron de TRR se encontraban en manejo con asistencia mecánica para respirar, mientras que sólo $0.2 \%$ de los aquejados no ventilados necesitaron diálisis. De los pacientes que requirieron VMI y que desarrollaron LRA, en $52.2 \%$ de ellos esto sucedió dentro de las primeras 24 horas postintubación. La mediana de tiempo de inicio de la VMI al inicio de TSR fue de 0.3 horas (RIC: $-41.1,92.3$ ). ${ }^{13}$

Aún falta mucho por conocer sobre la participación nefrítica en COVID-19, sin embargo, ésta parece implicar un proceso complejo y multifactorial. Mediado por daño viral directo, liberación de citocinas, activación de angiotensina II, alteraciones del complemento, hipercoagulabilidad y 
afección microangiopática; interactuando con los factores de riesgo clásicos de la aparición de deterioro de riñón, a saber: hipoperfusión, sepsis, SIRA, uso de nefrotóxicos, ventilación asistida, hipoxia de la médula renal y rabdomiólisis. 3,7,8,14-19

La interacción entre la actividad de angiotensina II, la respuesta inmune innata y adaptativa y las vías del complemento y el sistema de coagulación podrían influenciar la severidad y el desenlace en LRA asociada a COVID-19. ${ }^{14}$

El principal sitio de unión para el SARS-CoV-2 es la enzima convertidora de angiotensina dos (ACE2), altamente expresada a nivel renal, en el borde en cepillo del túbulo proximal. ${ }^{14,16}$

La replicación viral a nivel podocitario y el deterioro secundario podrían ser la causa de la proteinuria reportada en infectados con ese virus. Por otra parte, la activación de macrófagos y la micronagiopatía también podrían causar LRA y daño podocitario. ${ }^{14}$

Los pacientes con ERC, especialmente aquellos con nefropatía diabética, que desarrollan COVID-19, pueden estar en mayor riesgo de LRA por el aumento en la expresión basal de ACE y disminución de ACE2, combinación que promueve un estado proinflamatorio y profibrótico a nivel renal. ${ }^{14}$

Datos de autopsias indican afección del endotelio pulmonar y del riñón, probablemente responsables de la proteinuria. Un estudio histopatológico de seis autopsias encontró necrosis tubular aguda (NTA) severa con infiltrado linfocitario y de macrófagos, prominente daño agudo al túbulo proximal, agregación peritubular de eritrocitos, trombos de fibrina a nivel glomerular con colapso isquémico y detrimento endotelial. ${ }^{3,14}$

Partículas virales se han reportado en células endoteliales renales, indicando viremia como posible raíz del estrago del endotelio y contribución a la LRA. Adicionalmente, el SARS-CoV-2 puede infectar directamente ese epitelio tubular y podocitos a través de la ACE2 y provocar disfunción mitocondrial, NTA, glomerulopatía colapsante y fuga de proteínas de la cápsula de Bowman. ${ }^{3}$

También se ha observado la presencia de la proteína de nucleocápside del SARS-CoV-2 en estructuras tubulares y cuerpos de inclusión positivos a esta nucleocápside en el citoplasma. ${ }^{14,16}$

Su y su equipo observaron la presencia de partículas virales a nivel podocitario y tubular por microscopia electrónica, al igual que una tinción positiva para el anticuerpo contra la proteína nuclear del SARS-CoV-2 en el epitelio tubular. El SARS-CoV-2 podría infectar directamente los túbulos nefríticos e inducir inclusiones citoplasmáticas a este nivel, como se ha observado en otras nefropatías virales, aunque esto aún es controversial. ${ }^{14,15}$

La presencia de proteinuria subnefrótica y hematuria microscópica es consistente con los hallazgos en autopsias de necrosis tubular aguda con infiltrado de macrófagos del túbulo-intersticio y daño difuso tubular proximal con agregados de eritrocitos obstruyendo los capilares peritubulares. ${ }^{8,16}$

La tormenta de citocinas, o síndrome de liberación de citocinas, puede ocurrir en diferentes condiciones, incluyendo sepsis y síndrome hemofagocítico. En tal escenario la LRA puede ser resultado de inflamación de riñón, aumento en la permeabilidad vascular, depleción de volumen y síndrome cardiorenal. ${ }^{17}$ Este síndrome incluye detrimento endotelial sistémico, manifestado por derrame pleural, edema, hipertensión intraabdominal, fuga de líquido al tercer espacio, depleción intravascular e hipotensión. ${ }^{17}$

La interleucina 6 (IL-6) es la principal implicada y sus niveles plasmáticos están aumentados en aquellos con SIRA secundario a COVID-19. El uso de VMI, oxigenación por membrana extracorpórea (ECMO) y las terapias continuas de reemplazo renal (CRRT) también pueden contribuir a la generación de citosinas. ${ }^{17}$

También se ha demostrado la predisposición a trombosis, coagulación intravascular diseminada (CID) e infartos pulmonares en estos pacientes. Lo mismo que la evidencia de microangiopatía trombótica en otros órganos. Por lo que es posible que el estado hipercoagulable promueva la evolución de NTA a necrosis cortical, con el consiguiente maltrato renal irreversible. ${ }^{14}$ La presencia de agregados eritrocitarios a nivel peritubular y en capilares glomerulares sugiere el rol de la hipercoagulabilidad. ${ }^{15}$

En cuanto a la comunicación entre órganos, existe una relación cercana entre el daño alveolar y tubular en SIRA, revelado previamente por estudios en los que se ha observado el desarrollo de LRA en $68 \%$ de los involucrados con SIRA secundario a neumonía y es bien conocido que el SIRA puede provocar hipoxia en la médula renal. ${ }^{17}$

Más avanzada edad, severidad de la enfermedad, DM y balances de líquidos positivos se asocian con el surgimiento de la LRA. Adicionalmente, la seriedad de la LRA se liga positivamente con una mayor edad, IMC elevado, DM, falla cardiaca, PEEPs más altos y puntajes de Sequential Organ Failure Assessment (SOFA) más altos. ${ }^{12-17}$ Además, niveles elevados de IL-6 en LRA se han conectado a un aumento de la permeabilidad alveolo-capilar y a hemorragia pulmonar. ${ }^{17}$

Al hablar del eje corazón-riñón, se ve que el desarrollo de cardiomiopatía y miocarditis viral aguda puede contribuir a la congestión venosa, hipotensión e hipoperfusión nefrítica. El síndrome cardiorrenal, particularmente la falla cardiaca derecha secundaria a neumonía, puede provocar congestión en los riñones y LRA. De manera similar, la disfunción del ventrículo izquierdo con disminución del gasto cardiaco y del volumen arterial efectivo provocan hipoperfusión a este nivel..$^{3,17}$ 
Sin mencionar que el balance positivo de líquidos tiene un efecto deletéreo en SIRA, aumenta la fuga alveolocapilar y en LRA incrementa la congestión venocapilar, provocando síndrome compartimental renal. Por otro lado, el deterioro endotelial, la fuga a tercer espacio e hipotensión, provocan hipoperfusión renal. ${ }^{17}$ También se ha documentado la presencia de cilindros de pigmentos en autopsias de pacientes con elevación de creatinina fosfocinasa (CPK), sugiriendo rabdomiólisis como causa posible de la toxicidad tubular renal. ${ }^{14,17}$

La incidencia reportada de LRA asociada a COVID-19 tiene un rango muy amplio, dependiendo del país, definición de LRA utilizada, proporción de enfermos y características clínicas de la población bajo investigación.

En la mayoría de los estudios, la LRA se produce a lo largo de la hospitalización, en promedio de los cinco a nueve días posteriores al internamiento. Es más frecuente en aquellos pacientes críticos, con SIRA severo, con ventilación mecánica invasiva (VMI), edad más avanzada, hipertensos o diabéticos. ${ }^{6}$

Es posible que la incidencia reportada hasta el momento esté infraestimada, tanto por el diseño retrospectivo de los reportes, como por la falta de una definición operacional de LRA; a pesar de que la generalidad de los análisis utilizó la definición de KDIGO, pocos reportan el estadio de la LRA. Factores como raza, características demográficas, severidad de la enfermedad, tratamiento hemodinámico, uso de asistencia mecánica para respirar y nefrotóxicos, también podrían explicar la discrepancia entre los estudios.

Hasta la fecha de la realización de este manuscrito, no se han publicado reportes de incidencia, severidad, factores de riesgo, o desenlace de la lesión aguda de riñón en contagiados con SARS-CoV-2 en México. El objetivo de los autores es describir la aparición de semejante daño en pacientes hospitalizados por COVID-19 de moderado a grave, en un hospital privado de la Ciudad de México. Además, describir las particularidades clínicas de estos enfermos, el grado de seriedad de la lesión nefrítica aguda y el número de personas que requirieron terapia de sustitución de riñón como parte del manejo.

\section{MATERIAL Y MÉTODOS}

Se trata de un estudio observacional, descriptivo, transversal y retrospectivo. En su realización se revisaron los expedientes de los pacientes hospitalizados en el Hospital Ángeles Mocel (HAM) que ingresaron con diagnóstico de COVID-19 corroborado o sospechoso, en el periodo comprendido entre el $1^{\circ}$ de marzo y el 31 de julio del 2020.

Se incluyeron enfermos con diagnóstico de COVID-19, ratificado por reacción en cadena de polimerasa por transcriptasa reversa (RT-PCR) positiva para SARS-CoV-2, obtenida por hisopado nasofaríngeo, que contaran con expediente clínico en el HAM, mayores de 18 años de edad. También se incluyeron pacientes con diagnóstico de ERC grados uno a cuatro de KDIGO.

Por otra parte, se excluyeron enfermos que no contaran con prueba RT-PCR positiva para SARS-CoV-2, obtenida por hisopado nasofaríngeo, quienes permanecieron hospitalizados por menos de 48 horas, que no contaran con expediente clínico en el HAM o cuya información fuera incompleta, al igual que pacientes con ERC grado 5 de KDIGO o en tratamiento crónico de sustitución renal.

Este estudio fue aprobado por los comités de Investigación y de Ética en Investigación del Hospital Ángeles Mocel, y durante su elaboración no se realizó ninguna intervención.

Se revisaron los expedientes de todos los pacientes en el periodo antes mencionado. Los autores elaboraron una base de datos utilizando hojas de cálculo en Microsoft Excel, donde se recopilaron los pormenores demográficos, biométricos, comorbilidades, días de estancia hospitalaria, requerimiento de hospitalización en unidad de cuidados intensivos, laboratorios al ingreso y durante la hospitalización, prueba RT-PCR para SARS-CoV-2, requerimiento de oxígeno suplementario, necesidad de ventilación asistida, requerimiento de terapia de reemplazo renal con hemodiálisis y número de sesiones otorgadas, así como motivo de egreso. La recolección y análisis de datos se llevó a cabo por los autores en el periodo comprendido entre agosto y diciembre de 2020.

El diagnóstico de caso COVID-19 confirmado se estableció de acuerdo al "Lineamiento estandarizado para la vigilancia epidemiológica y por laboratorio de la enfermedad respiratoria viral", de la Secretaría de Salud de Méxi$\mathrm{Co}^{20}{ }^{20}$ como aquel con prueba de reacción en cadena de polimerasa por transcriptasa reversa obtenida por hisopado nasofaríngeo positiva para SARS-CoV-2.

El dictamen de lesión aguda de riñón y su clasificación se establecieron de manera retrospectiva de acuerdo a la clasificación de las guías KDIGO 2012, ${ }^{10}$ definiendo dicho estrago como un aumento de la creatinina sérica de al menos $0.3 \mathrm{mg} / \mathrm{dL}$ dentro de 48 horas o aumento de la creatinina sérica mayor o igual a 1.5 veces su valor basal en un periodo de siete días. En los pacientes que contaran con una cifra de creatinina en los siete a 365 días previos a su ingreso, esa se consideró como su valor basal. En los que no tuvieron creatinina basal en los siete a 365 días previos se conjeturó una creatinina para una tasa de filtración glomerular estimada de 75 $\mathrm{mL} / \mathrm{min} / 1.73 \mathrm{~m}^{2}$.

La severidad del daño renal se definió de la siguiente manera, de acuerdo a las guías KDIGO 2012: ${ }^{10}$

- Grado 1: aumento absoluto de la creatinina sérica de al menos $0.3 \mathrm{mg} / \mathrm{dL}$ dentro de 48 horas o aumento de 
Figura 1: Selección de la población de estudio. RT-PCR = reacción en cadena de polimerasa por transcriptasa reversa, ERC G5 = enfermedad renal crónica grado 5 de KDIGO.

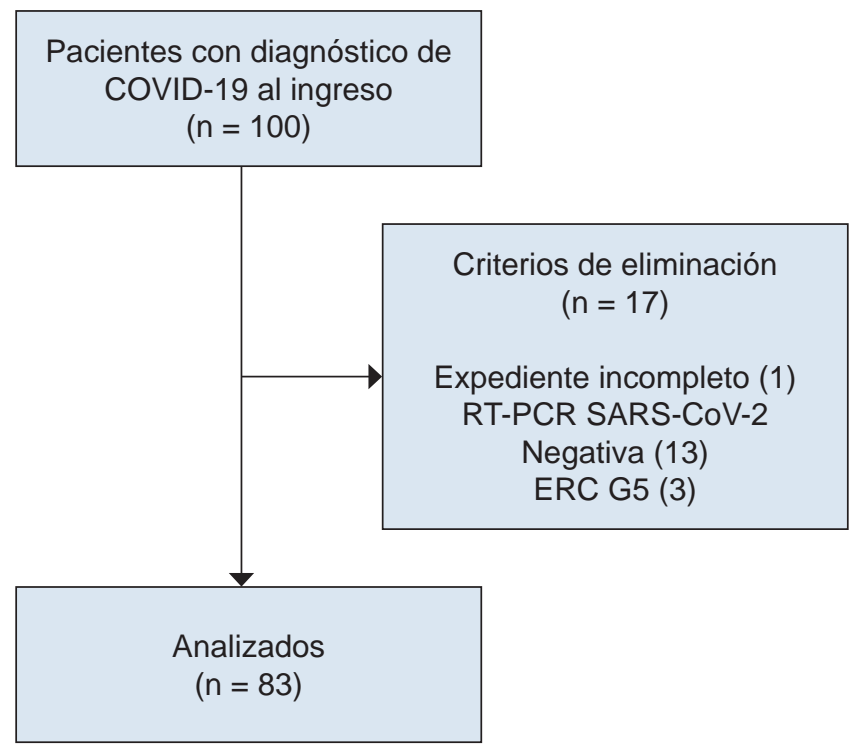

ésta de 1.5 a 1.9 veces su nivel basal en un periodo de siete días.

- Grado 2: incremento de la creatinina sérica de 2.0 a 2.9 veces su valor basal en un periodo de siete días.

- Grado 3: elevación de la creatinina sérica mayor o igual a 3.0 veces su cifra basal en un periodo de siete días, o medida de dicha creatinina igual o más grande que $4 \mathrm{mg} / \mathrm{dL}$, o bien inicio de terapia de reemplazo de la función de los riñones.

El momento de inicio de la terapia de sustitución renal con hemodiálisis convencional, al igual que la suspensión de la misma, fue decisión del médico nefrólogo a cargo de cada caso.

En el análisis estadístico de los datos se empleó el programa SPSS v.22 logrando el cálculo de rangos, porcentajes, medias, desviación estándar e incidencia.

\section{RESULTADOS}

Inicialmente, se incluyeron 100 pacientes con diagnóstico de COVID-19 al ingreso, sin embargo, luego se excluyeron 17 de ellos. De los excluidos, 13 no contaban con RT-PCR para SARS-CoV-2 positiva, tres del grupo tenían diagnóstico de ERC grado 5 de KDIGO y se encontraban en tratamiento con hemodiálisis de mantenimiento y un paciente no contaba con laboratorios de ingreso completos en el expediente. Así, se incluyeron 83 pacientes en el análisis final (Figura 1).
De los 83 aquejados incluidos en el análisis, 58 eran hombres $(69.8 \%)$ y 25 mujeres $(30.1 \%)$, en un rango de edad de 21 a 93 años, media de $54.66 \pm 18.17$ años. Las principales comorbilidades observadas fueron HAS (37.3\%), DM2 (22.8\%), dislipidemia (9.6\%), hipotiroidismo (6.0\%) y ERC (4.8\%).

El resto de las características clínicas y resultados de laboratorio se ilustran en las Tablas 1 y 2, respectivamente.

Del total de pacientes, 33 (39.7\%) ameritaron atención en $\mathrm{UCl}$, mientras que $50(60.24 \%)$ fueron manejados en área de hospitalización. El promedio de estancia hospitalaria fue de $12.12 \pm 7.10$ días, con un rango de tres a 41 días. La estancia en UCl fue de $9.24 \pm 8.13$ días con un rango de uno a 39 días, y en área de hospitalización fue de $9.56 \pm 3.74$ días con un rango de tres a 19 días.

Respecto al uso de oxígeno suplementario, 78 (93.9\%) requirieron algún tipo de apoyo. De éstos, 40 (48.1\%) se manejaron con puntas nasales convencionales, 15 (18.0\%) con puntas nasales de alto flujo (PNAF), 5 (6.0\%) con ventilación mecánica no invasiva (VMNI) y 18 (21.6\%) con la versión invasiva (VMI) con intubación orotraqueal.

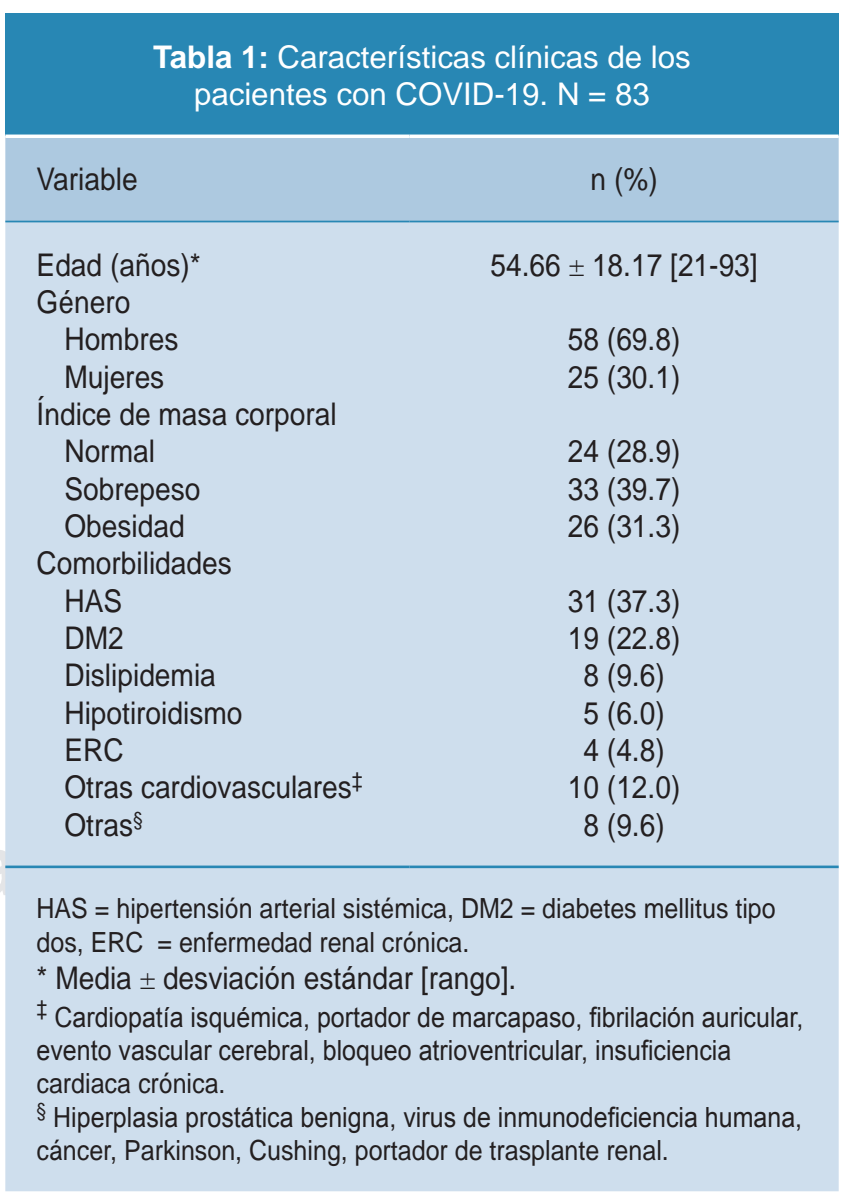


Tabla 2: Resultados de laboratorio al ingreso.

Parámetro de laboratorio

$\mathrm{Hb}(\mathrm{g} / \mathrm{dL})$

HTO (\%)

Plaquetas $\left(10^{3} / \mu \mathrm{L}\right)$

Leucocitos totales $\left(10^{3} / \mu \mathrm{L}\right)$

Neutrófilos absolutos $\left(10^{3} / \mu \mathrm{L}\right)$

Linfocitos absolutos $\left(10^{3} / \mu \mathrm{L}\right)$

BUN (mg/dL)

Urea (mg/dL)

Creatinina $(\mathrm{mg} / \mathrm{dL})$

TFG CKD-EPI mL/min/1.73m²

Sodio (mEq/L)

Potasio (mEq/L)

Cloro (mEq/L)

Calcio (mEq/L)

Fósforo $(\mathrm{mEq} / \mathrm{L})$

Dímero D (ng/mL)

Ferritina $(\mathrm{ng} / \mathrm{mL})$

Procalcitonina $(\mathrm{ng} / \mathrm{mL})$

Proteína C reactiva (mg/L)

Lactato $\mathrm{mmol} / \mathrm{L}$

$\mathrm{PaO}_{2} / \mathrm{FiO}_{2}$ (índice de Kirby)

m, [rango]

$$
\begin{aligned}
15.1 & \pm 1.1[18.7-7.9] \\
45.4 & \pm 5.53[24.9-57.3] \\
223.9 & \pm 76.9[94-483] \\
9,261.4 & \pm 4,667.7[2,000-23,700] \\
7.38 & \pm 4.65[0.12-21.8] \\
1.32 & \pm 0.62[0.18-3.3] \\
17.3 & \pm 9.35[6.1-21.2] \\
37.1 & \pm 20.07[12.9-112.7] \\
1.0 & \pm 0.35[0.57-2.78] \\
84.1 & \pm 25.7[16-135] \\
137.7 & \pm 4.13[127-149] \\
4.1 & \pm 0.61[2.9-6.8] \\
104.4 & \pm 4.71[88-116] \\
8.2 & \pm 0.58[7.1-10.3] \\
3.1 & \pm 1.16[0.89-9.5] \\
1,149.5 & \pm 1,180.95[92-7,443] \\
1,225.6 & \pm 1,434.93[22.9-9,542] \\
0.62 & \pm 1.23[0.02-9.11] \\
143.1 & \pm 101.19[0.2-438.4] \\
1.8 & \pm 0.94[0.1-4.3] \\
229.4 & \pm 112.23[47.1-542]
\end{aligned}
$$

$\mathrm{HB}=$ hemoglobina, $\mathrm{HTO}=$ hematocrito, $\mathrm{BUN}=$ nitrógeno ureico en sangre, $\mathrm{TFG}=$ tasa de filtración glomerular, $\mathrm{CKD}-\mathrm{EPI}=\mathrm{Chronic}$ Kidney Disease Epidemiology Collaboration, $\mathrm{PaO}_{2}=$ presión parcial de oxígeno, $\mathrm{FiO}_{2}=$ fracción inspirada de oxígeno.

De los 83 enfermos, 30 (36.14\%) cursaron con lesión renal aguda. De ellos, 20 (66.6\%) se encontraban en UCI y $10(33.3 \%)$ en área de hospitalización. Quienes estaban hospitalizados en $\mathrm{UCl}$ presentaron un puntaje promedio de APACHE II al ingreso de $8.25 \pm 3.12$, comparado con un puntaje de $6.6 \pm 2.83$ de aquellos hospitalizados fuera de $\mathrm{UCl}$.

En cuanto a la clasificación de la LRA, 13 pacientes (43.3\%) se calificaron con KDIGO 1, con $6(20 \%)$ y 11 (36.6\%) como KDIGO 2 y KDIGO 3 respectivamente (Tabla 3). Del total de aquejados con LRA, 7 (23.3\%) precisaron terapia de reemplazo de riñón con hemodiálisis convencional, el promedio de sesiones de hemodiálisis requeridas fue 3.16 sesiones con un rango de una a 10, todos los que necesitaron hemodiálisis fallecieron.

Dentro del grupo de internados que requirieron ventilación mecánica invasiva, 14 desarrollaron LRA, que corresponde al $77.7 \%$ de esta población. Mientras que, de los pacientes $\sin \mathrm{VMI}, 15$ sufrieron la LRA, que corresponde al $25 \%$ de esta población.

El principal motivo de egreso fue por mejoría en $71.0 \%$ de los casos, mientras que el $28.9 \%$ fallecieron. Del total de defunciones, 18 (75\%) fueron en pacientes hospitalizados en $\mathrm{UCl}$, los seis restantes (25\%) en área de hospitalización.
De los 18 enfermos con VMI, 15 (83.3\%) fallecieron, contrastado con 7 (11.6\%) de los 60 sin intubación orotraqueal.

En el grupo de afectados con LRA, 17 (56.6\%) fallecieron, que corresponde al $70.8 \%$ de la mortalidad total. De los 14 pacientes con VMI y LRA, 13 (86.6\%) fallecieron, comparado con 4 (26.6\%) de los 15 pacientes sin VMI que padecieron LRA.

\section{DISCUSIÓN Y CONCLUSIONES}

En este estudio, la incidencia de lesión aguda del riñón en contagiados con COVID-19 fue de 36.14\%, similar a lo reportado en algunas cohortes de Estados Unidos y Europa. $\mathrm{Al}$ igual que en la bibliografía internacional, el desarrollo de tal trastorno fue más frecuente en los pacientes que se encontraban hospitalizados en la Unidad de Cuidados Intensivos y en aquellos que necesitaron VMI como parte del tratamiento, encontrándose en dicho subgrupo de pacientes un surgimiento superior al reportado previamente en otros países.

En cuanto a la severidad del daño renal, todos los casos clasificados con KDIGO 3 se presentaron dentro de $\mathrm{UCI}$ y más del $60 \%$ necesitó TRR. Mientras tanto, la mayoría 
de los pacientes tratados fuera de $\mathrm{UCI}$ presentaron menor grado de tal deterioro.

Respecto a la mortalidad, los autores encontraron que $56.6 \%$ de los internados con LRA fallecieron. Esto corresponde al $70.8 \%$ de las defunciones totales. Se observó más alta mortandad en aquellos enfermos que se encontraban en $\mathrm{UCl}$ y padecieron LRA; señalando que el total de pacientes que recibieron terapia de reemplazo de riñón fallecieron.

Estas observaciones probablemente se deban a que los pacientes en área de hospitalización presentaron menor severidad tanto de la LRA, como de la neumonía por SARS-CoV-2. Este grupo de enfermos no requirió tratamiento con vasopresores, ni VMI, la estancia hospitalaria fue más corta y probablemente recibieron menos fármacos nefrotóxicos, comparado con quienes estaban internados en UCl. Además, los dolientes con LRA manejados en $\mathrm{UCl}$, incluyendo esos que recibieron TRR, al

\begin{tabular}{|c|c|}
\hline \multicolumn{2}{|c|}{$\begin{array}{l}\text { Tabla 3: Características de la lesión renal } \\
\text { aguda en pacientes con COVID-19. }\end{array}$} \\
\hline Variable & $\mathrm{n}(\%)$ \\
\hline Total de pacientes con LRA & $30(36.14)$ \\
\hline Pacientes en $\mathrm{UCI}$ & $20(66.6)$ \\
\hline Pacientes fuera de UCI & $10(33.3)$ \\
\hline \multicolumn{2}{|l|}{ Severidad* } \\
\hline KDIGO 1 & $13(43.3)$ \\
\hline Atención en UCl & $5(38.5)$ \\
\hline Fuera de UCI & $8(61.5)$ \\
\hline KDIGO 2 & $6(20.0)$ \\
\hline Atención en $\mathrm{UCl}$ & $4(66.6)$ \\
\hline Fuera de UCl & $2(33.3)$ \\
\hline KDIGO 3 & $11(36.6)$ \\
\hline Atención en UCl & $11(100.0)$ \\
\hline Con TRR & $7(63.6)$ \\
\hline \multicolumn{2}{|l|}{ Terapia de reemplazo renal } \\
\hline Pacientes con requerimiento & $7(23.3)$ \\
\hline Número sesiones & $3.16 \pm 3.30$ \\
\hline \multicolumn{2}{|l|}{ Pacientes con ventilación mecánica invasiva } \\
\hline Total de pacientes con VMI & $18(21.6)$ \\
\hline Pacientes con VMI y LRA & $14(16.8)$ \\
\hline \multicolumn{2}{|l|}{ Desenlaces } \\
\hline Alta por mejoría & $13(43.3)$ \\
\hline Defunciones totales & $17(56.6)$ \\
\hline Defunción en pacientes con TRR & $7(100.0)$ \\
\hline Defunción en pacientes con VMI y LRA & $13(86.6)$ \\
\hline \multicolumn{2}{|c|}{$\begin{array}{l}\text { LRA = lesión renal aguda, UCI = unidad de cuidados intensivos, } \\
\text { KDIGO = Kidney Disease Global Outcomes, TRR = terapia de reem- } \\
\text { plazo renal, VMI = ventilación mecánica invasiva. } \\
\text { ×De acuerdo con la clasificación de las guías KDIGO } 2012 .\end{array}$} \\
\hline
\end{tabular}

ingreso presentaban puntajes de APACHE II más altos que aquellos con deterioro renal manejados fuera de la UCI. Se debe recordar que los internados en estado crítico tienden a estar expuestos a más factores de riesgo tendientes al desarrollo de lesión aguda de riñón, por ejemplo el uso de vasopresores a dosis altas, nefrotóxicos, hipoperfusión renal, sepsis, VMI con PEEPs altos, sobrecarga hídrica, entre otros.

Por otro lado, los autores encontraron una cifra de decesos más elevada en dichos enfermos con VMI. Por tomografía, estos pacientes presentaban mayor porcentaje de afección pulmonar, la LRA fue más frecuente en ese subgrupo y el total de pacientes que recibieron TRR se encontraban bajo VMI. Estos hallazgos son similares a lo reportado en la bibliografía internacional, dejando claro que es probable que exista una relación entre la severidad de la LRA, estancia en $\mathrm{UCl}$ y el riesgo de presentar desenlaces adversos en COVID-19.

Ventajas del estudio: es del conocimiento de los autores que, hasta el momento de la redacción de este manuscrito, no se ha publicado ningún estudio que describa los rasgos clínicos generales de los pacientes con COVID-19 en población mexicana, ni la incidencia de lesión renal aguda en pacientes con esa enfermedad viral en área de hospitalización y en terapia intensiva en un hospital privado de la Ciudad de México.

Se observó que, en pacientes mexicanos hospitalizados por COVID-19, la aparición de ese daño renal agudo es más elevada en aquellos que necesitaron manejo en Unidad de Cuidados Intensivos y/o ventilación artificial. Al igual que mayor mortandad en quienes cursan con esa dolencia aguda durante su estancia en la $\mathrm{UCl}$, lo cual coincide con lo reportado hasta el momento en la literatura internacional.

Limitantes del estudio: Al ser un estudio de carácter retrospectivo no es posible tener control sobre las variables estudiadas. Además de que se contó con una muestra pequeña de pacientes.

Si bien se observó mortalidad incrementada en aquellos pacientes en $\mathrm{UCl}$ que desarrollaron LRA, no fue posible determinar si el aumento de decesos está relacionado de forma independiente con el desarrollo de daño del riñón. Es probable que la severidad de la LRA pueda ser un factor de riesgo independiente de mortandad en COVID-19 y vale la pena estudiarlo más a fondo.

Es necesario hacer estudios controlados aleatorizados comparando la mortalidad en pacientes con COVID-19 y ventilación mecánica invasiva, con y sin lesión renal aguda.

También es necesario explorar la relación entre el tiempo de inicio de dicha asistencia mecánica con el desarrollo de ese tipo de trastorno y el requerimiento de la terapia de sustitución de riñón. 


\section{REFERENCIAS}

1. Chaolin Huang, Yeming Wang, Xingwang Li, Lili Ren, Jianping Zhao, Yi Hu et al. Clinical features of patients infected with 2019 novel coronavirus in Wuhan, China. Lancet. 2020; 395 (10223): 497-506.

2. Zhu N, Zhang D, Wang W, Li X, Yang B, Song J et al. A novel coronavirus from patients with pneumonia in China, 2019. N Engl J Med. 2020; 382 (8): 727-733.

3. Ronco C, Reis T, Husain-Syed F. Management of acute kidney injury in patients with COVID-19. Lancet Respir Med. 2020; 8 (7): P738742.

4. Hassanein M, Radhakrishnan Y, Sedor J, Vachharajani T, Vachharajani V, Augustine J et al. COVID-19 and the kidney. Cleveland Clinic Journal of Medicine. 2020; 87 (10): 619-631.

5. Cheng Y, Luo R, Wang K, Zhang M, Wang Z, Lei Dong L. Kidney disease is associated with in-hospital death of patients with COVID-19. Kidney Int. 2020; 97 (5): 829-838.

6. Gabarre P, Dumas G, Dupont T, Darmon M, Azoulay E, Zafrani L. Acute kidney injury in critically ill patients with COVID-19. Intensive Care Med. 2020; 46 (7): 1339-1348.

7. Sise ME, Baggett MV, Shepard JAO, Stevens JS, Rhee EP. Case 172020: a 68-year-old man with COVID-19 and acute kidney injury. N Engl J Med. 2020; 382 (22): 2147-2156.

8. Pelayo J, Peterson E, Albano J, Patarroyo-Aponte G, Rangaswami J, Bryan Lo K et al. Clinical characteristics and outcomes of community and hospital acquired acute kidney injury with COVID-19 in a US inner city hospital system. Cardiorenal Med. 2020; 10 (4): 223-231.

9. Chan L, Chaudhary K, Saha A, Chauhan K, Vaid A, Baweja M et al. Acute kidney injury in hospitalized patients with COVID-19. medRxiv. 2020.

10. Khwaja A. KDIGO clinical practice guidelines for acute kidney injury. Nephron Clin Pract. 2012; 120 (4): 179-184.
11. Yih-Ting Chen, Shih-Chieh Shao, Cheng-Kai Hsu, I-Wen Wu, MingJui Hung, Yung-Chang Chen. Incidence of acute kidney injury in COVID-19 infection: a systematic review and meta-analysis. Critical Care. 2020; 24: 346.

12. Pei G, Zhang Z, Peng J, Liu L, Zhang C, Yu C et al. Renal involvement and early prognosis in patients with COVID-19 pneumonia. J Am SoC Nephrol. 2020; 31 (6): 1157-1165.

13. Hirsch JS, Ng JH, Ross DW, Sharma P, Shah HH, Barnett RL et al. Acute kidney injury in patients hospitalized with COVID-19. Kidney Int. 2020; 98 (1): 209-218.

14. Batlle D, Soler MJ, Sparks MA, Hiremath S, South AM, Welling PA et al. Acute kidney injury in COVID-19: emerging evidence of a distinct pathophysiology. J Am Soc Nephrol. 2020; 31 (7): 1380-1383.

15. Hassanein M, Thomas G, Taliercio J. Management of acute kidney injury in COVID-19. Cleve Clin J Med. 2020.

16. Ronco C, Reis T. Kidney involvement in COVID-19 and rationale for extracorporeal therapies. Nat Rev Nephrol. 2020; 16: 308-310.

17. Perico L, Benigni A, Remuzzi G. Should COVID-19 concern nephrologists? why and to what extent? The emerging impasse of angiotensin blockade. Nephron. 2020; 1-9.

18. Ali H, Daoud A, Mohamed MM, Salim SA, Yessayan L, Baharani J et al. Survival rate in acute kidney injury superimposed COVID-19 patients: a systematic review and meta-analysis. Renal Fail. 2020; 42 (1): 393-397.

19. Harrison P. COVID-19 and the kidney: another target to attack. Medscape. 2020.

20. Lineamiento estandarizado para la vigilancia epidemiológica y por laboratorio de la enfermedad respiratoria viral. Secretaría de Salud del Gobierno de México, Abril 2020.

Conflicto de intereses: Los autores declaran que no tienen ningún conflicto de intereses. 